\title{
MXT instrument on-board the French-Chinese SVOM mission
}

\author{
Karine MERCIER* ${ }^{1}$, François GONZALEZ ${ }^{1}$, Diego GÖTZ², \\ Martin BOUTELIER ${ }^{1}$, Narjiss BOUFRACHA ${ }^{1}$, Vadim BURWITZ ${ }^{4}$, Marie Claire CHARMEAU ${ }^{1}$, \\ Paul DRUMM ${ }^{3}$, Charlotte FELDMAN ${ }^{3}$, Albert GOMES ${ }^{1}$, Jean-Michel LE DUIGOU ${ }^{1}$, \\ Norbert MEIDINGER ${ }^{4}$, Aline MEURIS ${ }^{2}$, Paul O'BRIEN ${ }^{3}$, Julian OSBORNE ${ }^{3}$, \\ Pierre PASQUIER ${ }^{l}$, Laurent PERRAUD, James F. PEARSON ${ }^{3}$, Frédéric PINSARD $^{2}$, \\ Estelle RAYNAL ${ }^{1}$, Richard WILLINGALE ${ }^{3}$ \\ ${ }^{1}$ CNES: Centre National d'Etudes Spatiales (Toulouse, France) \\ ${ }^{2}$ CEA/Irfu - Département d'Astrophysique/AIM (Gif-sur-Yvette, France) \\ ${ }^{3}$ University of Leicester (Leicester United Kingdom) \\ ${ }^{4}$ Max-Planck-Institut für Extraterrestrische Physik (Garching Germany)
}

\begin{abstract}
SUMMARY
The SVOM (Space-based multi-band astronomical Variable Objects Monitor) French-Chinese mission is dedicated to the detection, localization and study of Gamma Ray Bursts (GRBs) and other high-energy transient phenomena. We first present the general description of the French payload composed of the ECLAIRs instrument, dedicated to GRB detection and localization and the MXT instrument, dedicated to GRB follow-up observation in soft X-ray band. Then the paper describes more in detail the design and the performances of the MXT instrument, finally a status of MXT development will be given.
\end{abstract}

\begin{abstract}
:
The SVOM (Space-based multi-band astronomical Variable Objects Monitor) is a mission developed within a SinoFrench cooperation context and dedicated to the detection, localization and study of Gamma Ray Bursts (GRBs) and other high-energy transient phenomena. Four instruments, sensitive in different wavelengths, employed on board to perform GRB detection and observations are accommodated on the Payload: they are (1) the trigger camera, called ECLAIRs, which triggers on the GRB present within its field of view (FOV) with accuracy of a few arc min ( 10 arc min) in the hard X-ray energy range (4 $\mathrm{keV}$ to $150 \mathrm{keV})$, (2) the gamma-ray spectro-photometer, called GRM (Gamma Ray Monitor), which observes simultaneously a larger field of view in the gamma-ray energy range, (3) the narrow field of view $\left(\sim 1^{\circ}\right) X$-ray telescope instrument, called MXT, which can quickly observe the error-boxes provided by ECLAIRs in the energy range $0.2 \mathrm{keV}$ to $10 \mathrm{keV}$ and (4) the narrow field-of-view visible telescope instrument, called VT, which can quickly observe the SVOM GRB afterglows in the optical band, and locate them to the arc sec precision.

At first, this paper will describe the French payload, developed under CNES responsibility in collaboration with French and European laboratories, composed of the ECLAIRs and MXT instruments. Then focus will be made on the MXT Instrument, an innovative focusing X-Ray telescope, compact and light (<35 kg) with a $1.15 \mathrm{~m}$ focal length whose concept is based on the coupling of 25 square 40 microns micro-pore optics (MPOs) with an actively cooled (-65 $\left.{ }^{\circ} \mathrm{C}\right)$ pnCCD (made of $256 \times 256$ pixels of 75 microns). Its architecture, its development and its expected performances will be presented. Thereafter, as the SVOM project Phase C/D has been started since one year, the MXT instrument manufacturing of first models are in progress so that preliminary results of tests on real subsystem like Optics, Focal Plane or electronic units can be presented. Finally we will give as a conclusion the main steps which have already been achieved and the further work to be accomplished in order to fulfill the MXT requirements and deliver the instrument to the satellite in 2020.
\end{abstract}

\section{INTRODUCTION}

SVOM (Space-based multi-band astronomical Variable Objects Monitor) is a space based astronomy mission complemented with ground-based dedicated instrumentation. The SVOM mission is devoted to the understanding of the origin and of the physics of the Gamma-Ray Bursts (GRBs) phenomena and other high-energy transient phenomena (Xray bursts, soft gamma repeaters, Active Galactic Nuclei (AGN), novae...). 
The SVOM mission is implemented by the China National Space Administration (CNSA), the Chinese Academy of Science (CAS) and the Centre National d'Études Spatiales of France (CNES); the SVOM mission is considered as a major part of the Chinese-French cooperation in the space domain.

In this paper, we first present the MXT instrument, inside payload, its architecture, its preliminary design, and the logic retained for its development. At the end, we will present a brief status of in progress manufacturing and some breadboard models results.

\section{THE SVOM MISSION}

The Gamma-ray bursts (GRBs) are highly variable and powerful cosmological explosions appearing randomly in the sky and characterized by very short (from a few milliseconds to hundreds of seconds) $\mathrm{X}-\mathrm{\gamma} \gamma$-ray flashes (the prompt emission) followed by a long-lasting afterglow emission observed from radio (up to several years later) to X-rays (up to several weeks after the $\gamma$-ray burst). Thus the SVOM mission was conceived with the Science objectives [1] to achieve a better understanding of the GRB phenomenon and other high-energy transient phenomena (X-ray bursts, soft gamma repeaters, Active Galactic Nuclei (AGN), novae...) given its related physics by providing a well characterized (spectrally and temporally) multi-wavelength dataset during the nominal 3 years mission lifetime extendable to 5 years.

The SVOM system (Figure 1) has been designed for early observation of the transient astronomical events - a key factor for the understanding of many fundamental physics questions- with a following observation strategy shared in several phases. At first, detection and localization of GRBs is performed by the SVOM instruments on the satellite then GRB data are sent to the ground in near real-time through a VHF stations network to enable ground based observations. At the same time, satellite can perform a slew to point at the GRB error box (if the gamma-ray signal level is high enough) and enabling observations by X-ray and visible narrow field optical telescopes.

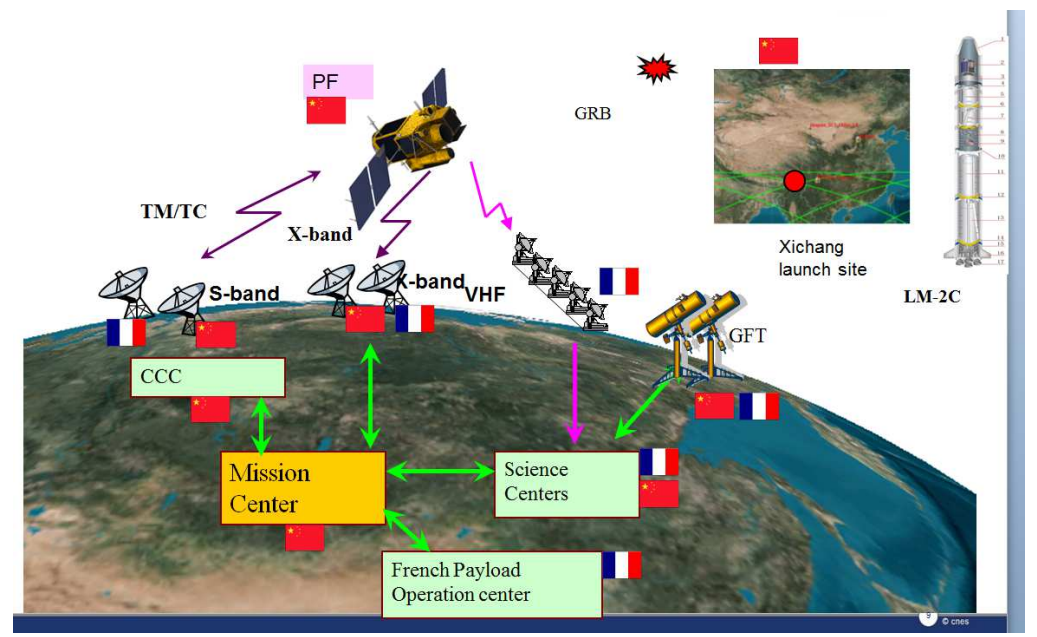

Figure 1: SVOM System overview

The instrumentation required for the SVOM mission is distributed between space (four instruments) see Figure 2 and ground. A combination of four multi-wavelength instruments is implemented on the satellite: ECLAIRs, a hard-X/soft- $\gamma$ ray imager and spectrometer, GRM, a $\gamma$-ray spectrometer, MXT, a soft X-ray telescope and VT, an optical telescope. On the other hand, on ground there are two 1-m class Ground Follow-up Telescopes (GFTs) located in Mexico and China and a set of GWACs (Ground Wide Angle Cameras) dedicated to the SVOM mission. The satellite orbit choice, altitude $\mathrm{h} \sim 625 \mathrm{~km}$ with an inclination about $28-30^{\circ}$ is adapted to the observation with these high-energy instruments where low level of the background is required.

To operate this complex system, it is essential to implement an uncommon operational concept driven by the mission requirements, and hence the SVOM level of on-board autonomy is higher than usual in order to modify and resume its activity program without any rescheduling activity from ground [3]. The SVOM observation plan mixes reactive observations of gamma-ray bursts (as soon as they are detected on-board) and observations of selected targets planned from ground once a week. Different priority levels are applicable to each category of observations. 


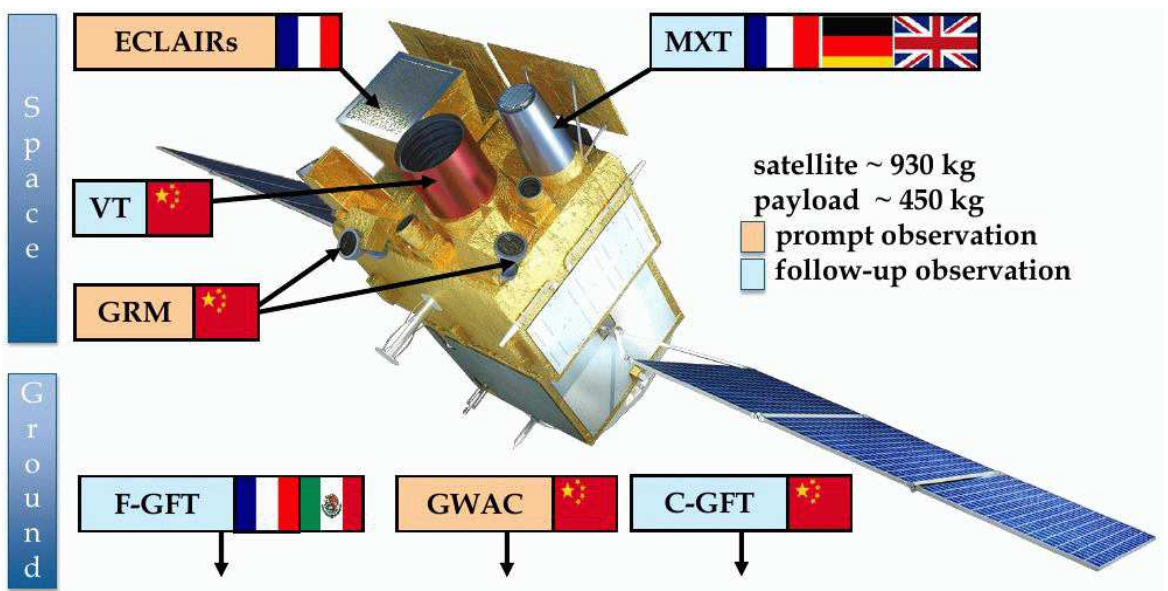

Figure 2: Satellite views in launch configuration (with SECM Courtesy)

To carry out this ambitious mission, SVOM is developed within the framework of a French-Chinese cooperation [2].

Thus, SVOM system responsibility is equally shared between CNSA/CAS and CNES, and the satellite is under CNSA/CAS responsibility; the platform and the integration of the satellite is under the responsibility of SECM (Shanghai Engineering Center for Microsatellites), the on-board payload is jointly provided by China and France, the launch services are under CNSA responsibility, with the choice of the LM2C launcher.

\section{THE SVOM SATELLITE AND PAYLOAD}

The necessary resources needed for this kind of mission lead to build a satellite in the small mission class; the orders of magnitude of the satellite mass and power are respectively $900 \mathrm{~kg}$ and $800 \mathrm{~W}$ with two three-sections rotatable solar panels. The overall satellite size is inside a circle of $2500 \mathrm{~mm}$ of diameter for $2800 \mathrm{~mm}$ height.

The platform design is based on a SECM platform (see Figure 3). It has strong heritage from their previous missions and re-used as much as possible existing units while meeting the specific requirements of the mission. On the other hand, the payload module design is specific to the SVOM requirements and allows accommodating all instruments.

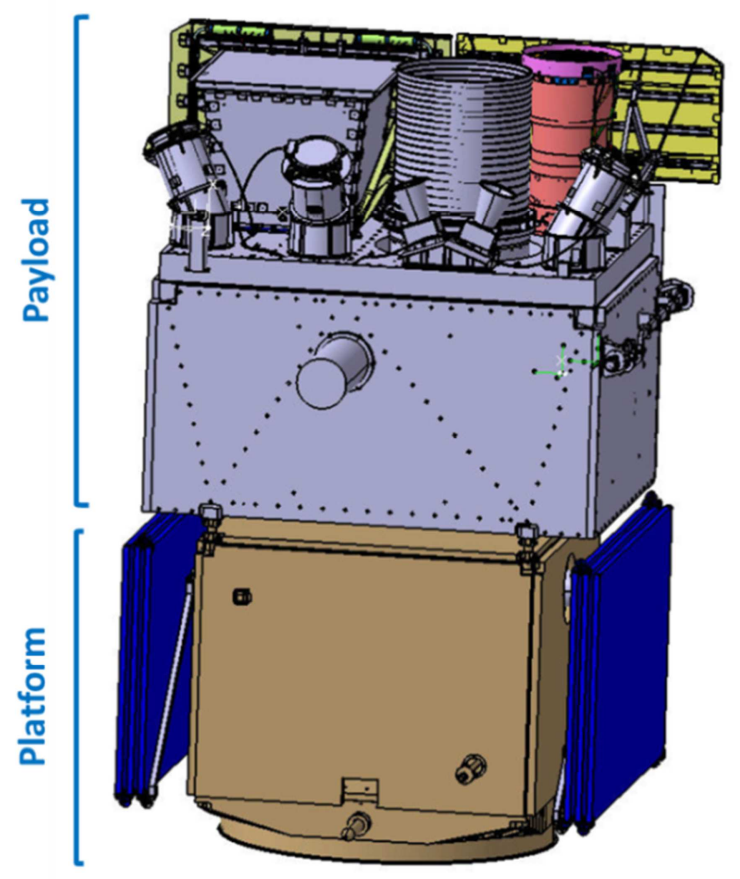

Figure 3: Satellite views in launch configuration (with SECM Courtesy) 


\subsection{The SVOM Payload Module (PLM)}

The payload Module (PLM) consists of a set of French and Chinese instruments, emitter systems (VHF-band, X-band) and On-board computer mounted upon a Payload Interface Module (PIM) which provides mechanical, electrical, and thermal support to the SVOM instruments (Figure 4).

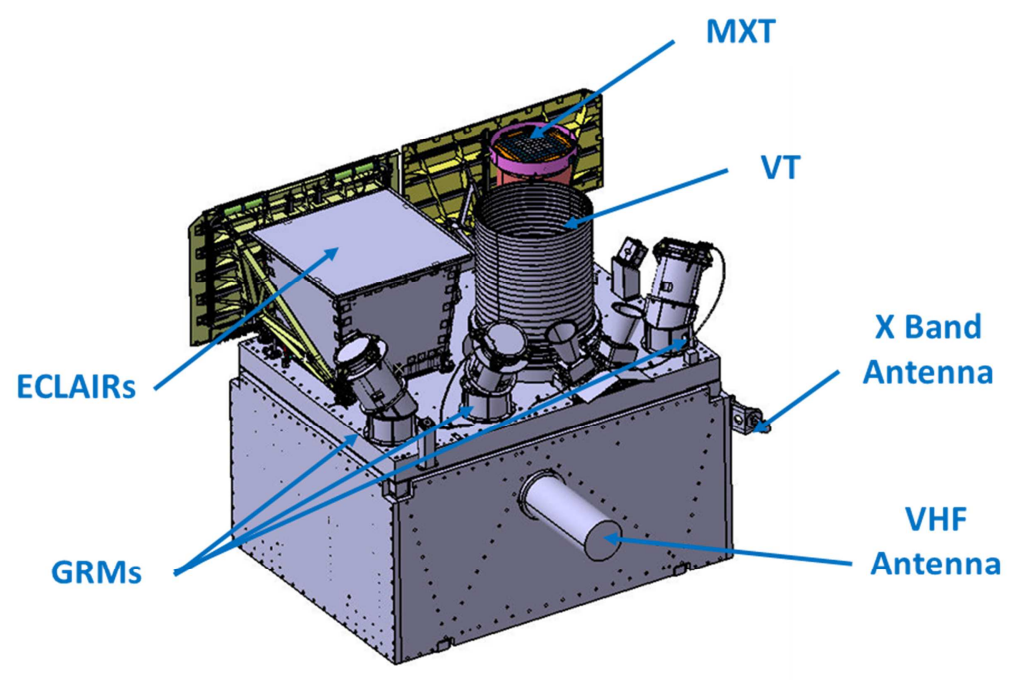

Figure 4 PLM External and Internal views

The two French instruments are:

- The ECLAIRs instrument dedicated to GRB detection and localization, having a large $(\sim 2$ sr $)$ field of view (FoV) and can detect GRBs in the hard X-ray energy band (4 keV to $250 \mathrm{keV}$ ). It can localize GRB in near real time with the position accuracy of a few arcmin ( 5-15 arcmin)

- The MXT instrument dedicated to GRB follow-up observation in the soft X-ray band (0.3 $\mathrm{keV}$ to $10 \mathrm{keV})$. The MXT, has a smaller FOV $\left(\sim 1^{\circ} \mathrm{x} 1^{\circ}\right)$ and can provide after satellite pointing towards the GRB, a localization better than 2 arcmin

The two Chinese instruments are:

- The VT (Visible Telescope) dedicated to GRB follow-up observation on board. The VT has relatively small FOV (26 arc min x 26 arc min), which needs satellite re-pointing to place GRB in this FOV. The VT instrument is composed of mechanical part and 2 camera heads $(400-650 \mathrm{~nm}$ and $650-1000 \mathrm{~nm})$, and also electronics for thermal control function. Its sensitivity $\left(22.5 \mathrm{M}_{\mathrm{V}}\right)$ is well adapted to detect the majority of the SVOM GRB afterglows.

- The GRM (Gamma Ray Monitor) spectrometer dedicated to the measurement of gamma-ray in the high energy band $(50 \mathrm{keV})$ to an upper threshold of $5 \mathrm{MeV}$. The hardware consists of three detectors, one CPM (Charged Particle Monitor) and correlative electronics. The CPM can give a message when the satellite passes through some place where too much particles gathering, such as SAA (South Atlantic Anomaly). Such message can inform the science instruments for protection and data management.

The payload emitter systems are:

- The VHF subsystem dedicated to transmit GRB messages to ground in real time. Burst data and some important instrument HK data can eventually be transmitted through it. This link is always active during satellite operations

- The X-Band Subsystem dedicated to scientific data download, which has interface with Payload computer.

The four instruments are controlled by the PDPU (Payload Data Processing Unit), a Chinese unit accommodated inside the payload module. It performs the general management of the overall instruments, the VHF alert, the scientific data storage and transmission. It also provides the platform interface for data handling. 


\section{MXT INSTRUMENT}

This X-ray instrument, under CNES responsibility is developed in collaboration with European laboratories. It is part of the new generation of focusing X-ray telescope whose concept is based on the "Lobster Eye" geometry. Thus MXT is a light and compact instrument compatible with the limited resources (volume, mass and power) available on the SVOM payload.

The design with a large field of view ( 1-degree side) and with a sensitivity below the mCrab level make of MXT a very good instrument to identify and precisely localize (below 2 arcmin) X-ray transients and to study them in detail, thanks to its excellent spectral response. It is designed to measure from 0.2 to $10 \mathrm{keV}$ with a maximum sensitivity around $1 \mathrm{keV}$. Then MXT will improve the GRB locations derived on board by ECLAIRs instrument.

MXT main specifications are presented in table below:

\begin{tabular}{|c|c|}
\hline \multicolumn{2}{|c|}{ Summary of MXT scientific requirement } \\
\hline Energy Range & $0.2-10 \mathrm{keV}$ \\
\hline Field of view & $57 \times 57 \operatorname{arc} \min$ \\
\hline Angular resolution & $<6.5 \operatorname{arc} \min ($ FWHM @ 1 keV) \\
\hline Source location accuracy & $\sim 100 \operatorname{arcsec}(90 \%$ c.l.) for a source at $5 \sigma$ \\
\hline Sensitivity $(5 \sigma)$ & 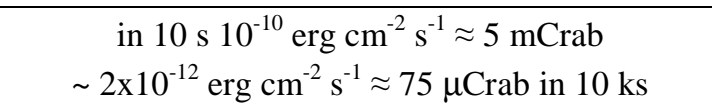 \\
\hline Throughput & $\begin{array}{c}1 \mathrm{mCrab} \sim 0.20 \mathrm{ct} / \mathrm{s} \text { for } \mathrm{N}_{\mathrm{H}}=4.5 \times 10^{21} \mathrm{~cm}^{-2}, \text { photon } \\
\text { index }=2.08\end{array}$ \\
\hline Energy resolution & 75 eV (FWHM @ 1 keV) \\
\hline Time resolution & $\leq 100 \mathrm{~ms}$ \\
\hline
\end{tabular}

\subsection{MXT project organization}

In the SVOM project, MXT is a project by itself, managed by CNES and involving many European partners and collaborators.

CEA Saclay having a large heritage in scientific focal planes is also in charge of the development of the Camera integrating the DUO pnCCD detector provided by the Max-Planck-Institut für Extraterrestrische Physik. The Leicester University already involved on Bepi-Colombo with a similar kind of optics and having experience with Micro-pore optics technology develops the optics of MXT. The LAL laboratory (Laboratoire de L'Accelérateur Linéaire) has the responsibility for the development of the scientific part of the on board instrument's software.

Obviously, all these institutes or laboratories take part in SVOM mission scientific issues and more specifically for MXT under the lead of the Principal Investigator (PI) of the MXT instrument D. Götz from CEA/Irfu.

At the end, with a large project team CNES is in charge of the system and architecture of the overall instrument. The integration and the validation of the whole MXT models will take place in Toulouse.

In addition, French or European companies have been chosen (after a call for tender) to manufacture several parts of the instrument. 


\subsection{MXT Architecture}

Today, the MXT project is in Phase C/D after its PDR (Preliminary Design Review) in September 2016 and the instrument architecture is now mature.

The instrument is shared in two parts, the telescope mounted on the top stable panel of the payload module while its electronic boxes are accommodated inside the Payload Module (see Figure 5).

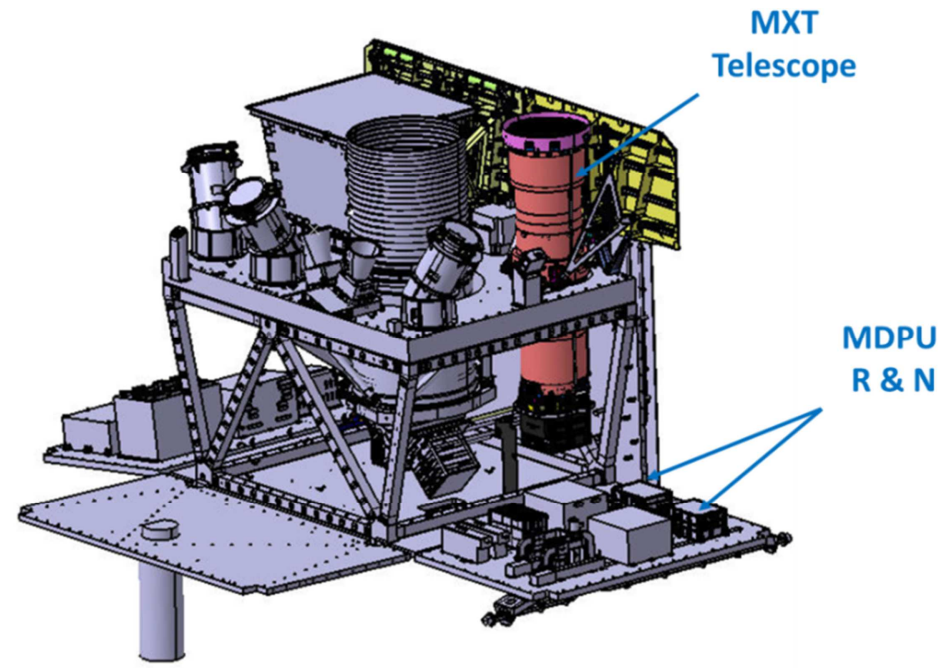

Figure 5: MXT inside Payload module

The instrument is designed to fulfill the SVOM mission requirements, especially to answer the MXT Science requirements and to the SVOM System requirements. Thus, the architecture is the result of all these considerations with the challenge of designing a stable instrument for localization performance, with a cooled $\left(-65^{\circ} \mathrm{C}\right)$ focal plane (for optimal performance and radiation damage reduction) in a very variable thermal environment (low Earth orbit and agile satellite) with always in mind the allocations in mass and power available on the satellite.

The telescope is composed by four main subsystems (Figure 6): an optical module, a camera, a carbon fiber structure, and a radiator. It has a nominal focal length of $1.15 \mathrm{~m}$.

The instrument effective area is a combination of the optics effective area, the transmission of the aluminum filters, the CCD quantum efficiency equal to $22 \mathrm{~cm}^{2}$.

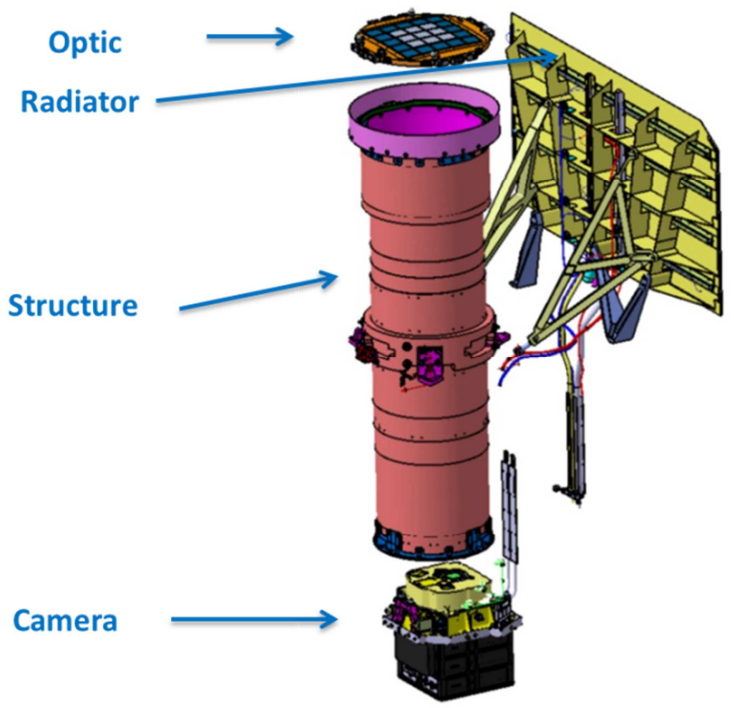

Figure 6: MXT telescope components 


\subsection{MXT Optics}

The optics of MXT is based on a "Lobster Eye" geometry and optimized for a narrow-field use. The X-rays hit the inner walls of the micro-pores with grazing incidence. The Point Spread Function (PSF) is composed by a central spot and two cross arms (Figure 7). The central spot is formed by rays with two reflections inside the MPO pores. The PSF arms correspond to rays with only one reflection. About $\sim 50 \%$ of the incident X-ray flux is focused in the central spot, $2 \mathrm{x}$ $\sim 20 \%$ in the arms, and the rest in a diffuse patch. Thanks to the "Lobster Eye" geometry the vignetting is very low, of the order of few $\%$ at the edge of the FOV.

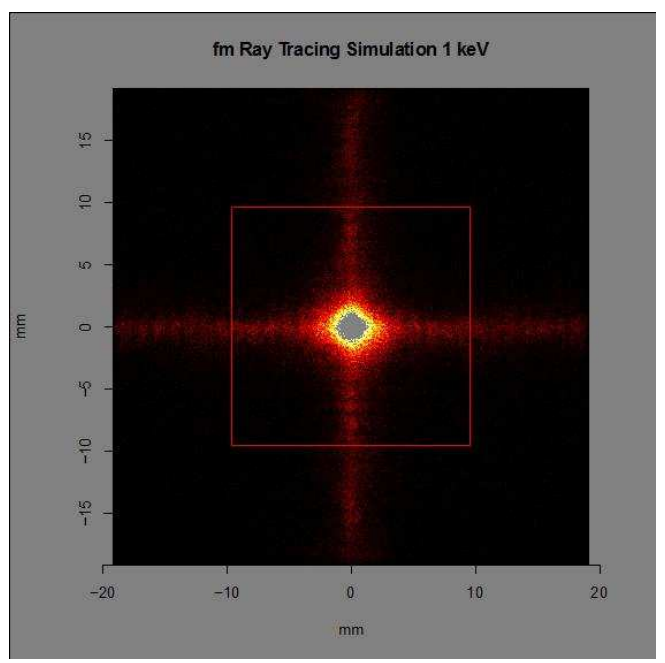

Figure 7: Shape of the PSF simulated

Since 2015, the University of Leicester in charge of the MXT Optics has developed a breadboard [4] to validate the optical design and the performance. The design is a compromise between the mechanical behavior, the thermal stability and of course the optical performance required for such an instrument.

The optic is composed of 25 micro-pore optics (MPO), manufactured by Photonis (in Brive France). The MPO plate of 40 x $40 \mathrm{~mm}$ size has $\sim 600000$ square pores with $\mathrm{d}=40 \mu \mathrm{m}$ size and a pitch of $\mathrm{p}=52 \mu \mathrm{m}$. The inner walls are coated with a $25 \mathrm{~nm}$ Ir layer to boost the reflectivity. The plate thickness is optimized to avoid vignetting and maximize the on-axis effective area. The central plates have $2.4 \mathrm{~mm}$ thickness while the outermost ones have $1.2 \mathrm{~mm}$. They are bonded on an aluminum frame which upper face is a sphere of radius $2300 \mathrm{~mm}$, with $10 \mu \mathrm{m}$ machining accuracy. The MPO are covered with a $70 \mathrm{~nm}$ Aluminum film to avoid thermal flux and straylight from entering the instrument. On the back side there is an electron diverter in self-cancelling array, with 36 magnets glued in a grid. It allows deflecting the electrons from the focal plane (see Figure 8).
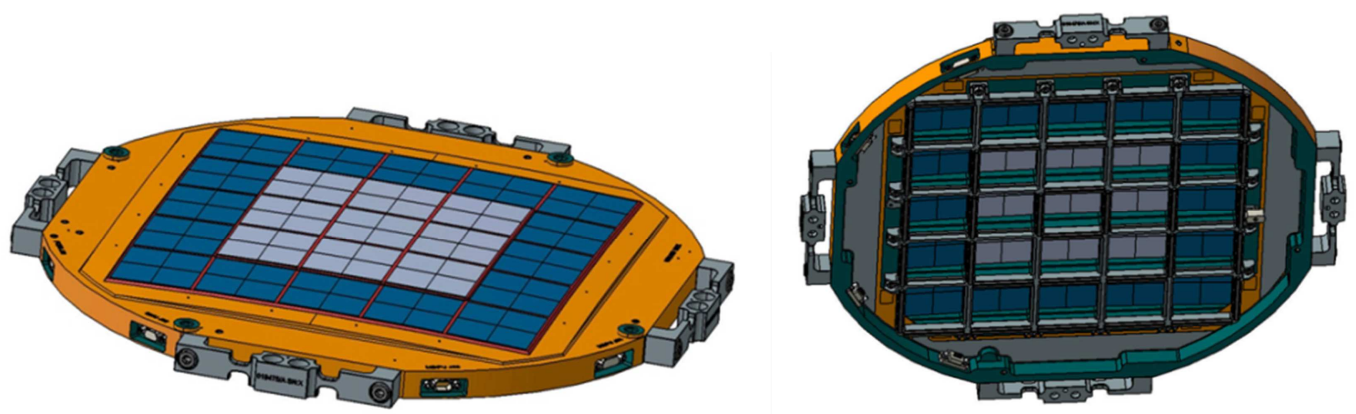

Figure 8: 25 MPOs on the aluminum frame (left), electron diverter on the back side (right)

The QM model is now in progress and will be tested before the end of 2018. It will allow confirming the expected performance and the space qualification at technological and optical subsystem level. In parallel the FM plates have just been delivered to UoL and individual testing will start next summer. 


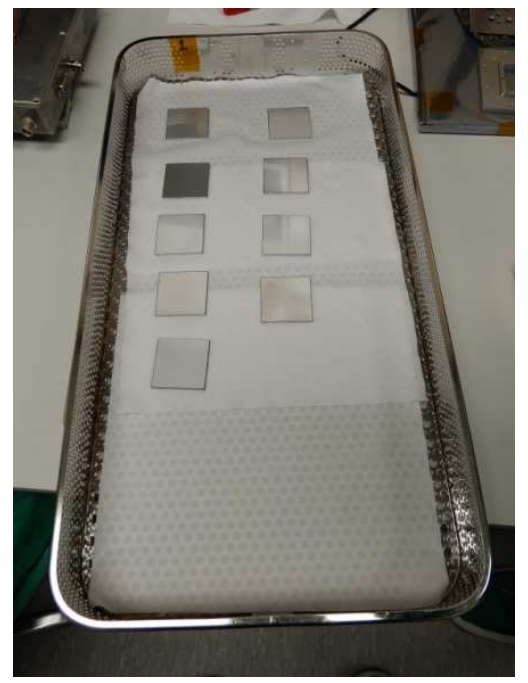

Figure 9: MPO (1,2 $\mathrm{mm})$ FM plates

\subsection{MXT Camera}

The MXT camera is the subsystem in which the pnCCD spectro-imaging sensor (256x256 pixels of $75 \mu \mathrm{m})$ associated with two 128-channel CAMEX front-end ASICs, provided by the Max Planck institute for Extraterrestrial Physics (MPE) is implemented. With a $1.15 \mathrm{~m}$ focal length, each pixel corresponds to about 13.5 arc sec. The FOV is limited by the CCD size and is a $57 \times 57$ arcmin square.

To ensure the optimal functioning of the $\mathrm{CCD}$ (and to limit the radiation damage), it is necessary to implement an active thermal system to keep it temperature below $-65^{\circ} \mathrm{C}$ (by 3 Thermal Electrical Cooler TEC), an aluminum shielding to limit radiation induced X-ray background and avoid CCD degradation and loss in charge transfer efficiency, a Front-End Electronic (FEE) to drive the detector and perform its readout data digitalization and finally a filter wheel (see Figure 10). Four positions are possible on the wheel, one open position for afterglows' observation, a position with an optical filter to be used for non-GRB science, a closed position with a radioactive source for energy calibration and a closed position with a copper shutter to protect the camera in the radiation belts and to perform dark current calibration.

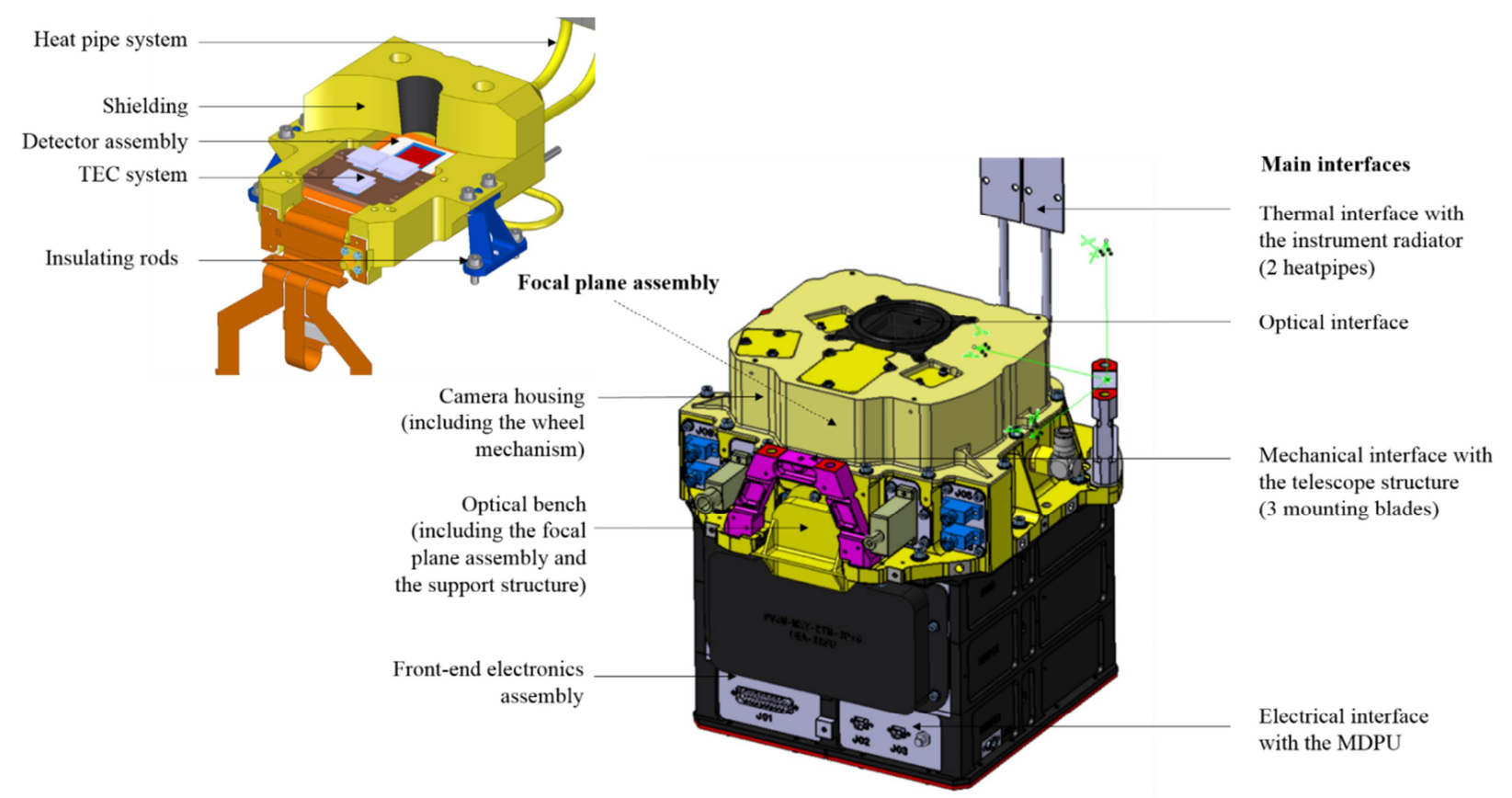

Figure 10: Camera design 
The mechanical and thermal design is a trade-off between a mass optimization and efficient shielding and cooling of the focal plane while withstand high mechanical levels coming from Satellite environment. The next step is to validate the concept and it mechanical and thermal behavior with the STM model in the coming months (Figure 11).

In the same way, in order to reach the Camera performances, the FEE design is finalized in order to be able to drive the CCD/CAMEX with the challenging specifications concerning the noise level and the timings of the readout sequence, using for the first time with this kind of detector non US electronic components. Several models EM, QM and FM will allow confirming and tuning the electronic design and performance.

The characterization of the whole focal plane (design and assembly CCD / CAMEX / ceramic) at the right temperature by the controlled by TEC is realized step by step. At first, the characterization of the TEC system, afterwards the CAMEX are bonded on the focal plane alone, then the FEE driving is tested and at the end the full chain.

The FEE EM model is under test, as the first focal plane model (BBM1 without CCD), full end to end tests are planned before the end of the year 2018 (Figure 11). The Camera full qualification and characterization will be realized with the QM model which will be manufactured in 2019.
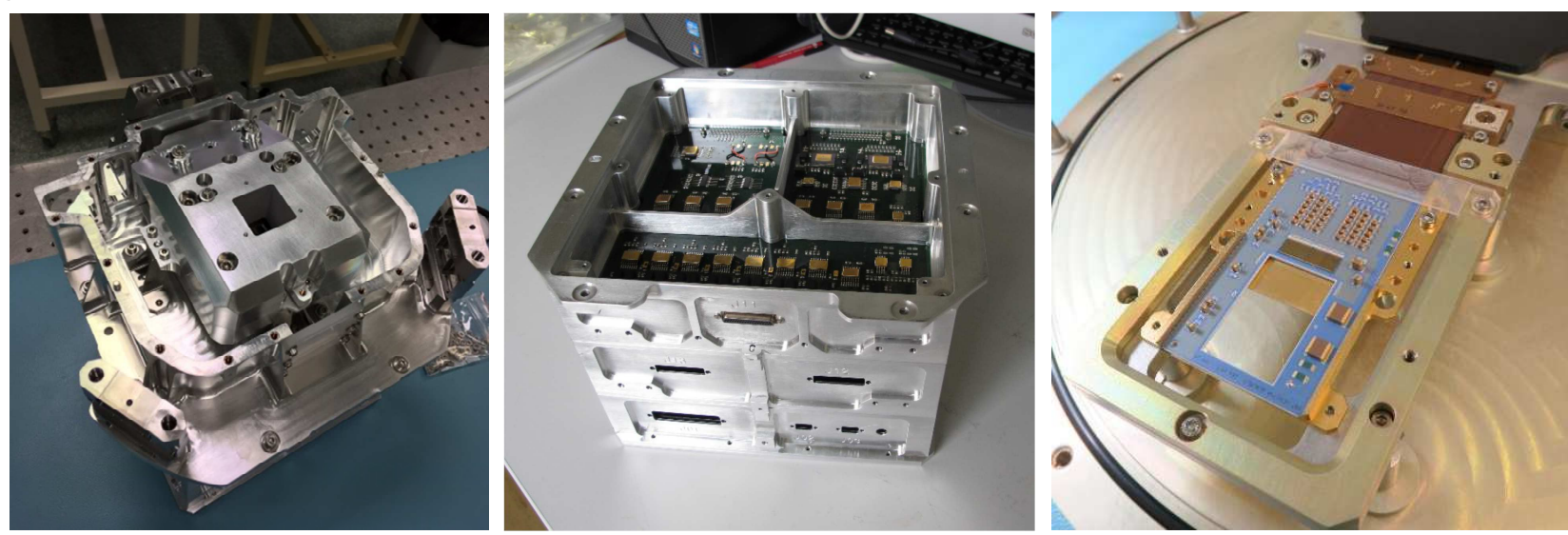

Figure 11: Camera STM (left) / FEE EM model (center) / Focal Plane: Detector assembly with 2 Camex (right)

\subsection{MXT Structure}

The MXT structure should support the Optic, the Camera and realize the external interface with the payload Module. Challenge for this element is to optimize design at first to withstand SVOM mechanical environment - while minimizing mechanical environment at sub-systems levels (Camera, Optic)-, to have high rigidity with mass as reduced as possible and then to realize and maintain telescope alignment throughout the lifetime of the mission.

The Critical Design Review has been successfully passed, and the proposed design fulfilled to requirements and thus this authorizes the first model manufacturing.

The MXT Structure consists of a CFRP tube (manufactured in only one piece), with on the middle three Titanium legs fixed at $120^{\circ}$, at the bottom and at the top titanium rings to interface with the Optic and the Camera. Choice of CRFP and titanium rings has been driven by thermo-elastic consideration to maintain alignment of elements in the SVOM high variable thermal environment.

At the top at the Optic interface a machinable shim will allow to realize the good alignment between Optic, the camera and the position on the Payload interface.

In addition, a small baffle is fixed at the top providing a protection against direct sun illumination, and an interior radiative cone to ensure thermal control (by radiation) of the Optics (Figure 12). 


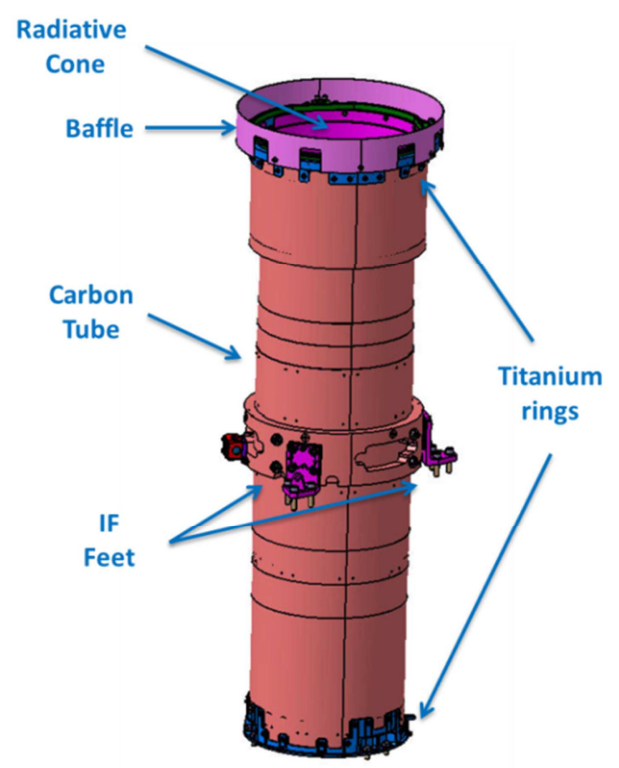

Figure 12: Structure design

The next model of the structure manufactured this autumn 2018 will allow integrating first MXT instrument model "STM (Structural and Thermal Model) which is required for the mechanical qualification and thermal characterization.

\subsection{MXT Radiator}

The MXT Thermal Control System has mainly to guarantee that the CCD shall be maintained at temperature $\leq-65^{\circ} \mathrm{C}$ in operations and a good stability and homogeneity of the telescope temperature. The challenge for the implementation of this thermal system comes because the environment of the mission is very unfavorable and because the available radiative surfaces are limited $\left(\sim 0.45 \mathrm{~m}^{2}\right.$ on the $-\mathrm{Zs}$ wall).

During phase $\mathrm{C}$ a new design of this radiator has been proposed reaching the requirement (Critical Design Review done see Figure 13), it is composed by six Propylene CCHP (Constant Caloduc Heat Pipe) with 2 of which connect directly with the heat pipes coming out of the camera.

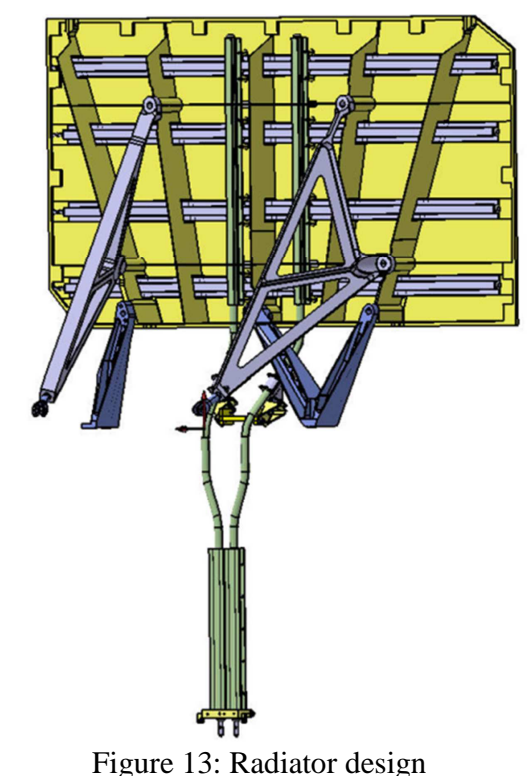

The first Radiator model is being built and will be integrated on the MXT telescope STM and then to be able to thermally characterize the system. 


\subsection{MXT Data Processing Unit - MDPU}

MXT Data Processing Unit (MDPU) is the electronic box in charge of driving the instrument and realizing the interface with the satellite. It is composed of two sub systems, a hardware part, in charge of supplying the power supplies, managing the external and internal interfaces at the hardware level, and providing a computing capacity and scientific processing of the data and a software system consisting of an operating system and command / control services.

Two identical MPDU boxes are installed on the payload module panel in order to realize a cold redundancy of the system.

\subsubsection{Hardware $M D P U$}

MDPU hardware is designed to be able to:

- Realize the interface with the satellite power distribution box (EPD) which provides the primary power supply of MXT;

- Carrying out the interface with the PDPU payload module calculator through the management of the Spacewire link and the management of the PPS synchronization signal;

- Generate, provide and sequence the switching on and off of secondary power supplies to the camera proximity electronics (MCAM/FEE);

- Provide hardware control functions for temperature control, temperature acquisition and controlled supply of heaters and cooling system;

- Interface with camera proximity electronics (MCAM) through command and control link management and acquisition of telemetry signals and photon data;

- $\quad$ Acquire the position and control the camera wheel (MCAM);

- Provide sufficient computing and working memory capabilities to host the flight software and enable real-time execution of scientific data processing algorithms whose GRB fine localization.

Resulting from Preliminary Design Review done in June 2017, the MDPU architecture (Figure 14) is finalized to a five boards architecture layout inside the box. One CPU board which contains among other Leon 3 CPU (GR712), one ACQ board which contains an I/O FPGA with ADC to support thermal regulation of TEC, to control secondary voltage power supply, to manage MDPU HK acquisitions, and three DC/DC converter boards which distribute power supply to MXT sub-systems (secondary voltage for the FEE and focal plane, motor power supply, TEC power supply).

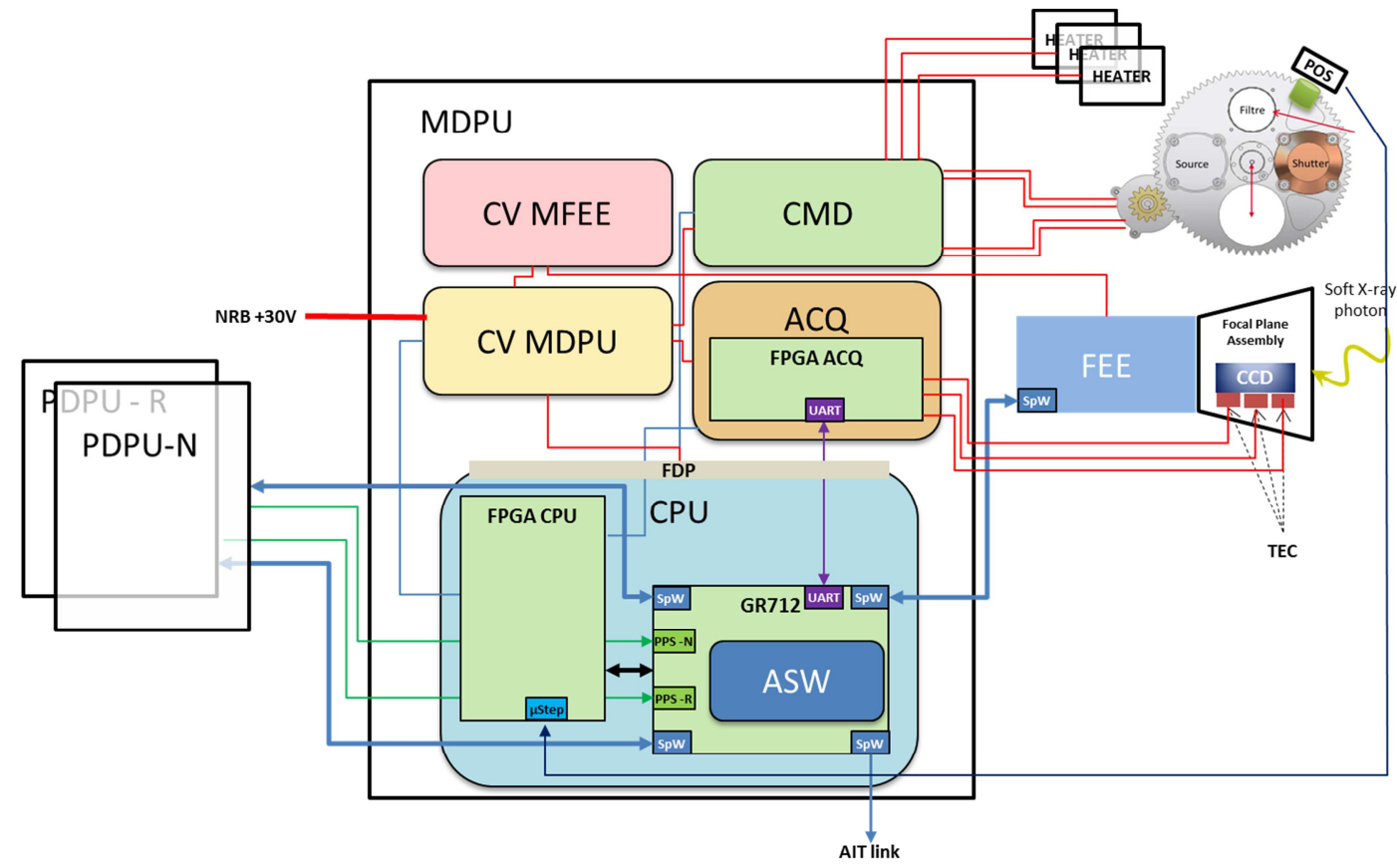

Figure 14: MDPU general architecture 
The first model MDPU BBM2, in form and fully representative but with lower level quality of components, has been delivered, it is dedicated to first functional and performance tests. The next model used for the full qualification of the unit (EQM model) is in progress. Feedbacks is expected for the end of this year in order to start flight model (FM), it will be thus available end 2019 to be able to start integration activities on instrument FM model.

\subsubsection{MDPU Applicative software}

For its development the MXT software should consider at the same time requirements coming from the satellite and needs from the instrument itself.

The MXT software is composed of two parts of system software: a Boot software which is responsible for initialization code executed at hardware reset, system self-tests, Boot report generation, loading and executing Application Software (ASW); an ASW which supports the following functions (Figure 15) in its nominal mode of execution:

- Command, control and monitor the MXT sub-system power supply

- Manage, control and monitor the PDPU interface (SpaceWire link, pulse per second (PPS) signal)

- Manage the different TM/TC flows including VHF, S band and X band

- Manage functional modes

- Manage the Data Handling interface with the FEE through a SpaceWire link (FEE configuration, scientific and housekeeping data acquisition)

- Manage the Camera mechanism (motor driving and wheel control)

- Manage the thermal control and regulation

- Manage the data processing in different modes

- Manage the monitoring and FDIR (Fault Detection and Isolation) of MXT internal hardware and software

The ASW receives from the PDPU (Chinese Payload Data Processing Unit) SpaceWire interface all telecommands and some messages coming from the platform software (attitude and orbit information, time relative to last PPS). Using the APID, these data are then routed by a dedicated function ("Input message router") which is in charge of distribution of input messages to Broadcasted data extraction function (attitude, orbit and time messages) and to TM/TC management component (telecommands).

Most of the functionalities of the MXT ASW are controlled by the software mode management function. This function is responsible for all mode transitions, from the verification of initial conditions, the software functions configuration and activation up to the verification of final conditions and the emission of on board events. It also manages FDIR that triggers mode transition. Depending on the transition that has been triggered, this function will activate attitude and orbit processing function, switch ON/OFF camera secondary voltages, activate the control of wheel motor, configure and activate the thermal control, configure and activate data acquisition and data processing, configure the FEE.

All scientific data processing from the source localization up to the camera calibration are contained in the data processing component. This part is a dedicated development under the LAL laboratory responsibility. 


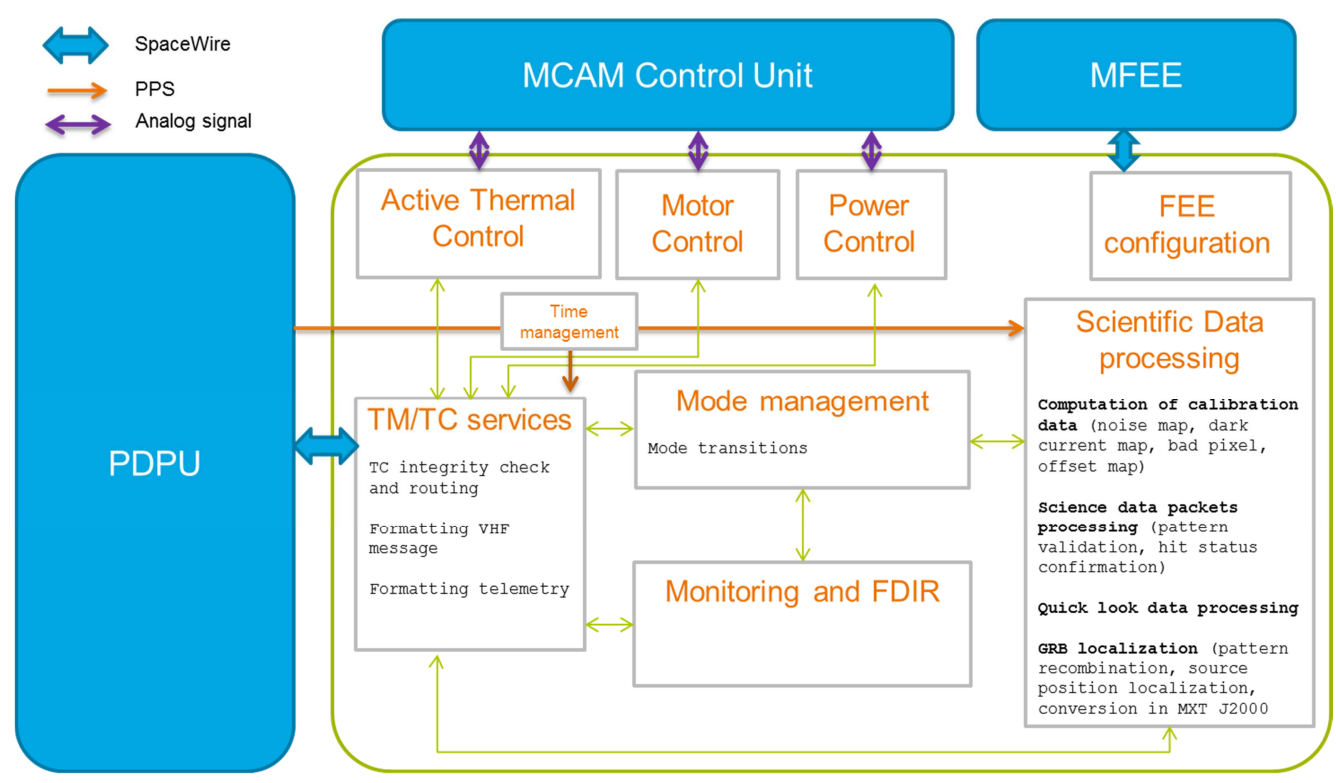

Figure 15: Applicative software functional scheme

The first version of the ASW has been delivered to CNES and implements the following functions that will be used at satellite test level (QM satellite campaign):

- Command, control the MXT sub system power supply (TEC, FEE, Heater)

- Manage, control and monitor the PDPU interface (SpaceWire link, PPS signal)

- Manage the different TM/TC flows including VHF, S- band and X- band

- Manage functional modes

- Manage the thermal control and regulation

It is currently under test in CNES facilities on board MDPU BBM2 model hardware (Figure 16) allowing to validate these functions before delivery to China.

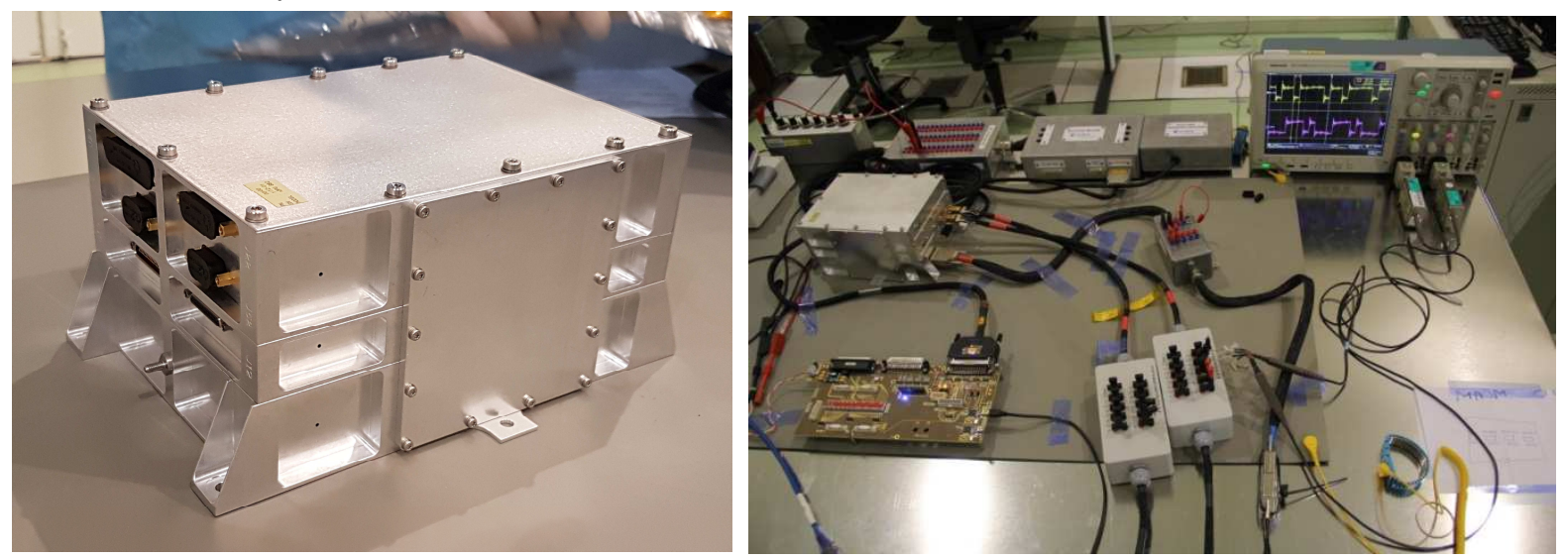

Figure 16: MDPU BBM2 under testing at CNES

The ASW will be completed with remaining functions for mid-2019; to be able to run end to end tests with MDPU EQM and Camera QM models to assess MXT performances. 


\section{MXT INSTRUMENT DEVELOPEMENT}

The MXT project proposes a development and validation policy resulting from the best compromise considering technical, cost and calendar criteria to optimize the number of developed models. The baseline is to develop and deliver a Proto Flight Model. However, to be in accordance with satellite development and validation plan, two additional models will be developed, a Structural and Thermal Model of the telescope and an Engineering and Qualification Model of the management and processing unit.

At first a Structural and Thermal Model (STM) of the telescope (MTEL) allowing to support mechanical qualification and thermal validation at instrument and satellite levels is to be manufactured.

In parallel an Engineering and Qualification Model (EQM) of the MDPU is manufactured in order to qualify this unit (mechanical/thermal/EMC) and validate software integration inside the hardware to be able at the end to support coupled test with satellite on the QM satellite.

At the end a Proto-Flight Model of MXT instrument (MDPU, MTEL and internal harnesses) will be realized with full validation plan before delivery, including mechanical, thermal and electrical tests (qualification level, acceptance durations), functional tests, performance tests and calibration.

This development logic is transposed at subsystem level. Nevertheless, it will be adapted to the sub-systems if necessary, with additional models due to their specific needs, according to their criticality, complexity, level of maturity. In any case these additional models will be used to limit some identified risks.

Today the MXT team at CNES and at laboratories works hard to manufacture the telescope STM model and the MDPU EQM model for autumn 2018 to validate the design of the instrument.

Since April 2018, a test campaign has started at CNES with the goal of validating the MDPU BBM2 model (first hardware and software version) using all dedicated functional test benches developed for this project.

Following these validations, CNES will deliver these models to SECM for satellite integration. Indeed, a large test campaign with a QM (qualification model) satellite will be conducted from mid-2018 to mid-2019, functional tests with the entire satellite avionics chain will be done in the first time on a "flatsat" then all elements will be integrated on a full representative satellite to perform the whole qualification tests (mechanical, thermal and EMC).

In addition, MXT project develops a "System Validation bench" in order to:

- Support functional and system validation of the delivered software

- Generation of typical data products used for ground data processing validation

- Train for operations

This simulator is fully representative of the MXT instrument command control function, it is composed of models of the platform (PCDU, PDPU) of the MDPU Hardware (LEON3 emulator) and of Camera (FEE, Motor, TEC). It can be interfaced with the French SVOM ground segment (French Science Center, French Payload Operation Center, MXT Instrument Center).

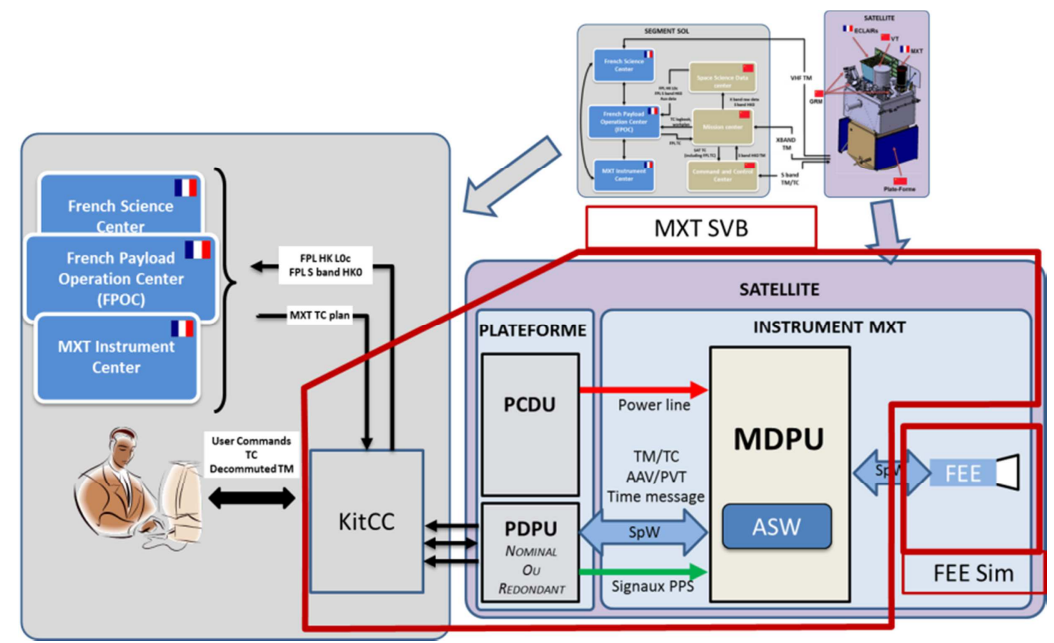

Figure 17: MXT System Validation Bench scheme

The first version will be available end 2018, with a goal for final version end of 2019. 


\section{CONCLUSION}

Phase C / D is well engaged, the MXT instrument and its sub systems design is now detailed. Several simulations already performed have shown that with this proposed architecture MXT is able reach the required specifications.

The coming months will witness a strong activity at all levels, as much from subsystem point of view than at instrument level. Different test results will validate technological choices made for the camera, the optics, the structure, the radiator and the MDPU. In addition, an important step for the project will be STM integration with manufactured subsystems to perform the mechanical qualification of the instrument with respect to the SVOM satellite environment and the thermal characterization next autumn.

Before PFM model, a required major step will be to analyze tests results at sub system, instrument and satellite level in order to adjust and improve if it is necessary the design of the instrument.

The work performed during previous years has allowed to pass important steps for MXT development but the following years will be challenging with the realization of real models.

\section{REFERENCES}

[1] J. Wei, B. Cordier et al., "The deep and transient Universe: New Challenges and Opportunities - Scientific Prospects of the SVOM Mission," arXiv: 1610.06892

[2] François Gonzalez, Shunjing YU, "SVOM: a French/Chinese cooperation for a GRB mission", SPIE (2018)

[3] Marie-Claire Charmeau et al., "SVOM payload mission operation concept " SpaceOps Conference, Marseille, France, 2018

[4] Charlotte Feldman, James Pearson, Richard Willingale, John Sykes, Paul Drumm, Paul, Houghton, Chris Bicknell, Julian Osborne, Adrian Martindale, Paul O'Brien, Ray Fairbend, Emile Schyns, Sylvain Petit, Romain Roudot, Karine Mercier, Jean-Michel Le Duigou, and Diego Gotz : "Testing and modelling of the SVOM MXT narrow field lobster eye telescope optic" SPIE 2017- 10399-61 